\title{
Positivity Preserving Interpolation of Positive Data by Rational Quadratic Trigonometric Spline
}

\author{
M.Dube ${ }^{1}$ and P.S.Rana ${ }^{2}$ \\ ${ }^{I}$ Department of Mathematics and Computer Science, R.D.University, Jabalpur, (M.P.) India. \\ ${ }^{2}$ Department of Engineering Mathematics, GGITS Jabalpur,(M.P.) India.
}

\begin{abstract}
In Computer Aided Geometric Design it is often needed to produce a positivity preserving curve according to the given positive data. The main focus of this work is to visualize the positive data in such a way that its display looks smooth and pleasant. A rational quadratic trigonometric spline function with three shape parameters has been developed. In the description of the rational quadratic trigonometric spline interpolant, positivity is preserved everywhere. Constraints are derived for shape parameters to preserve the positivity thorough positive data. The curves scheme under discussion is attained $C^{l}$ continuity.
\end{abstract}

Keywords: Interpolation, positivity, Rational quadratic trigonometric spline, Shape parameters.

\section{Introduction}

In recent years a good amount of work has been published (see [1], [2], [5], [6]) that focuses on shape preserving curves and surfaces. Positivity is an important shape preserving property. There are many physical situations where values are taken in positive sense only. For example, in a probability distribution, the presentation is always positive. Another application is in observation of gas discharge during certain chemical experiment are in process. Therefore, it is important to discuss positive interpolation to provide a computationally economic and visually pleasing solution to the problems of different scientific phenomena.

In this paper, we use a rational quadratic trigonometric spline in the description of positivity preserving of positive data. The significance of trigonometric spline in other areas, such as electronic and medicines is acknowledged in Hoschek and Lasser [7]. Recently trigonometric polynomials and trigonometric splines have gained some interest within Computer Aided Geometric Design. Schoenberg [8] has introduced the trigonometric B-spline in 1964. The cubic Bezier curve with two shape parameters introduced by Xi-An Han et al [9].

In this paper we examined the problems of shape preserving property of positive data, the cubic Hermite interpolation scheme ([10],page 102-106) is not better because the positive data in table $1 \mathrm{~s}$ interpolated by that scheme, the curve looks like not positive as shown in Hussain et al [11] of figure 1(a) and figure 2(a). For reducing this difficulty the authors use the rational function with four shape parameters. They preserved the positivity of positive data very well and looking very smooth as shown in the figures $1(\mathrm{~b}), 1(\mathrm{c})$, 2(b) and 2(c). In this paper, we use the alternative rational quadratic trigonometric spline for development of positivity preserving interpolation with three shape parameters. The trigonometric spline interpolation is easy to implement for $2 \mathrm{D}$ data and to compute very easily due to rational form of the function. It constructs $C^{1}$ interpolant and there is no additional points are inserted. The scheme developed in this paper is applicable when the derivatives are estimated by estimation technique which was given in Hussain [11]. The scheme of this paper is computationally less expensive than schemes in Hussain et al [11], Sarfraz et al [2] by using piecewise rational quadratic trigonometric function for the same purpose.

The discussion in this paper is arranged as follows: the definition of rational quadratic trigonometric spline is written in section 2. The determination of derivatives is discussed in section 3. The positivity preserving quadratic trigonometric interpolation given in section 4 . Some numerical examples and conclusion of the paper are discussed in section 5 and section 6 respectively.

\section{Rational Quadratic Trigonometric Function}

In this section, the rational quadratic trigonometric function with three shape parameters has been developed. Let $\left\{\left(t_{i}, f_{i}\right), i=0,1,2, \ldots n\right\}$ be the given set of positive data points defined over the interval [a, b], where $a=t_{0}<t_{1}<t_{2}<\cdots \ldots<t_{n}=b$. The $C^{1}$ rational quadratic trigonometric function with three shape parameters $\alpha_{i}, \beta_{i}$ and $\gamma_{i}$ are defined over each subinterval $\left[t_{i}, t_{i+1}\right], \mathrm{i}=0,1,2, \ldots, \mathrm{n}-1$ as:

$$
S(t)=S_{i}(t)=\frac{p_{i}(\theta)}{q_{i}(\theta)}
$$

Where 


$$
\begin{aligned}
p_{i}(\theta)=\alpha_{i} f_{i}(1- & \sin \theta)^{2}+\left(\frac{2 h_{i} d_{i} \alpha_{i}}{\pi}+\gamma_{i} f_{i}\right) \sin \theta(1-\sin \theta)+\left(\gamma_{i} f_{i+1}-\frac{2 h_{i} d_{i+1} \beta_{i}}{\pi}\right) \cos \theta(1-\cos \theta) \\
& +\beta_{i} f_{i+1}(1-\cos \theta)^{2}
\end{aligned}
$$

and

$$
q_{i}(\theta)=\alpha_{i}(1-\sin \theta)^{2}+\gamma_{i}(\sin \theta(1-\sin \theta)+\cos \theta(1-\cos \theta))+\beta_{i}(1-\cos \theta)^{2}
$$

Where $h_{i}=t_{i+1}-t_{i}, \theta=\frac{\pi\left(t-t_{i}\right)}{2 h_{i}}, 0 \leq \theta \leq \frac{\pi}{2}$ and $\alpha_{i}, \beta_{i}$ and $\gamma_{i}$ are free parameters. The equation (1) gives the rational quadratic trigonometric function of $C^{1}$ continuity, if following conditions are satisfies

$$
\begin{aligned}
P\left(t_{i}\right) & =f_{i}, P\left(t_{i+1}\right)=f_{i+1} \\
P^{\prime}\left(t_{i}\right) & =d_{i}, P^{\prime}\left(t_{i+1}\right)=d_{i+1}
\end{aligned}
$$

Where $P^{\prime}$ denotes the derivative with respect to t and $d_{i}$ denotes the derivative values at knotst $t_{i}$. The $\alpha_{i}, \beta_{i}$ and $\gamma_{i}$ are shape parameters preserved the positivity of the trigonometric interpolation with suitable constraints.

\section{Determination of Derivatives}

Usually, the derivative parameters $d_{i}$ are not known. They can be calculated either from the given data points $\left\{\left(t_{i}, f_{i}\right), i=0,1,2, \ldots n\right\}$ or by some other means. In this paper, $d_{i}$ 's are calculated from the given data points in such a way that the smoothness of the interpolant (1) is maintained. The following method is the approximation based on various mathematical theories. The description of such approximations is as follow:

\subsection{Arithmetic Mean Method [3]}

This method is three point difference approximation with

And the end conditions are given as

$$
d_{i}=\left\{\begin{array}{cl}
0 & \text { if } \Delta_{i-1}=0 \text { or } \Delta_{i}=0 \\
\frac{h_{i} \Delta_{i-1}+h_{i-1} \Delta_{i}}{h_{i}+h_{i-1}}, & \text { otherwise } i=2,3, \ldots n-1
\end{array}\right.
$$

$$
\begin{aligned}
& d_{1}=\left\{\begin{array}{cc}
0 & \text { if } \Delta_{1}=0 \\
\frac{\Delta_{1}+\left(\Delta_{1-} \Delta_{2}\right) h_{1}}{h_{1}+h_{2}}, & \text { otherwise }
\end{array}\right. \\
& d_{n}=\left\{\begin{array}{cr}
0, & \text { if } \Delta_{n-1}=0 \\
\frac{\Delta_{n-1}+\left(\Delta_{n-1}-\Delta_{n-2}\right) h_{n-1}}{h_{n-1}+h_{n-2}}, & \text { otherwise }
\end{array}\right.
\end{aligned}
$$

\section{Positivity Preserving Rational Quadratic Trigonometric Spline Interpolation}

The $C^{1}$ piecewise rational quadratic function (1) does not give guarantee to preserve shape of positive data, it is required to assign suitable values of shape parameter $\gamma_{i}$, then it gives a positive curve, to remove this deficiency some mathematical treatment is required which will be explained in the following way.

Let us assume positive data set $\left(t_{i}, f_{i}\right), i=0,1,2 \ldots$. n.

So that

$$
f_{i}>0
$$

In this paper, we would find sufficient and simple constraints for single shape parameter in order to preserve the positive curve of positive data in each sub interval if

$$
S_{i}(t)>0, \mathrm{i}=1,2, \ldots \mathrm{n} \text {. }
$$

The key idea is to determine constraints for $\alpha_{i}, \beta_{i}$ and $\gamma_{i}$ which guarantee to preserve the shape of positive data. The equation (4) is satisfies if both numerator $\&$ denominator of (1) are positive such that $p_{i}(\theta)>$

$$
0, q_{i}(\theta)>0
$$

Therefore the necessary condition for free parameters to preserve shape of positive data are

$$
\alpha_{i}>0, \beta_{i}>0 \text { and } \gamma_{i}>0
$$

We have $q_{i}(\theta)>0$ if equation (5) is satisfied and it only remains to show that numerator $p_{i}(\theta)$ in (1) is positive. $p_{i}(\theta)>0$ if

$$
\begin{aligned}
& \gamma_{i}>-\frac{2 h_{i} d_{i} \alpha_{i}}{\pi f_{i}} \\
& \gamma_{i}>\frac{2 h_{i} d_{i+1} \beta_{i}}{\pi f_{i+1}}
\end{aligned}
$$

The result in equation (6) and (7) can be summarized as 
Theorem 4.1 The $C^{1}$ piecewise rational quadratic trigonometric interpolant (1) defined over the interval [a, b] preserve the positivity of positive data in each subinterval if the sufficient conditions are satisfied.

$$
\gamma_{i}>\max \left\{0,-\frac{2 h_{i} d_{i} \alpha_{i}}{\pi f_{i}}, \quad \frac{2 h_{i} d_{i+1} \beta_{i}}{\pi f_{i+1}}\right\}
$$

\section{Numerical Example}

Table (1): Positive Data Set

\begin{tabular}{|l|l|l|l|l|l|l|l|l|}
\hline $\mathrm{i}$ & 1 & 2 & 3 & 4 & 5 & 6 & 7 & 8 \\
\hline$t_{i}$ & 1 & 2 & 3 & 8 & 10 & 11 & 12 & 14 \\
\hline$f_{i}$ & 14 & 8 & 3 & .8 & .8 & .45 & .40 & .37 \\
\hline
\end{tabular}

Figure 1(a) : Cubic Hermite Spline Interpolation

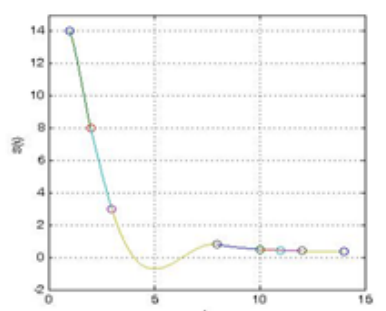

Figure 1(a) : Cubic Hermite Spline Interpolation

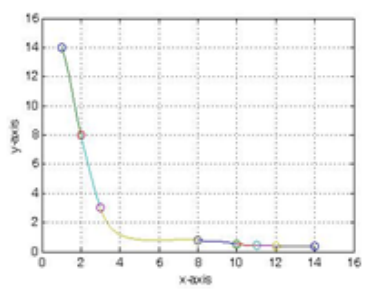

Figure 1(b) : Positive Rational Cubic Spline [9] with $r_{i}=w_{i}=.5$

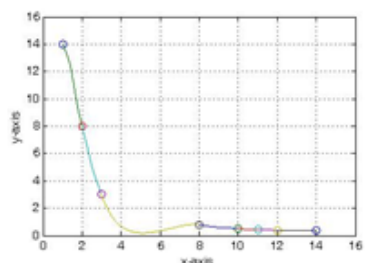

Figure 1(c) : Positive Rational Cubic Spline [9] with $r_{i}=w_{i}=2$

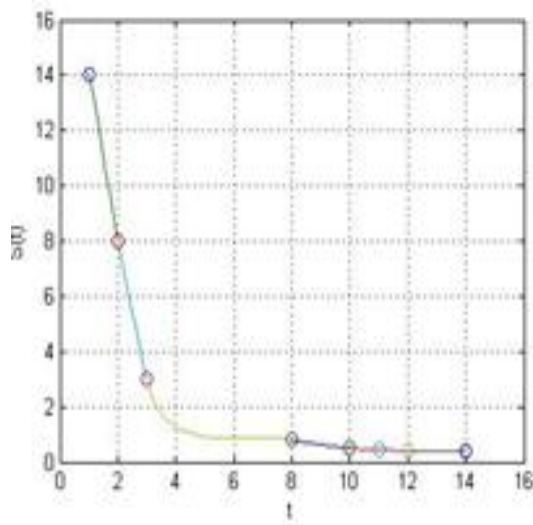

Figure 1(d): Rational Quadratic Trigonometric Spline interpolation with $\alpha_{i}=\beta_{i}=.1$ 


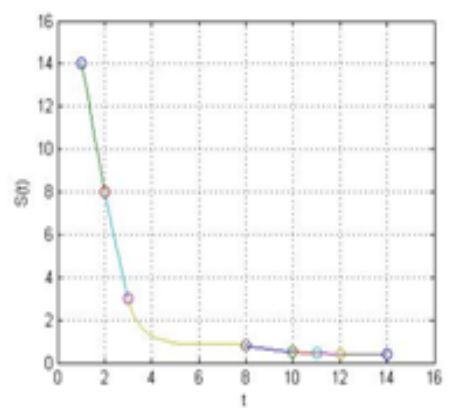

Figure 1(e) : Rational Quadratic Trigonometric Spline interpolation with $\alpha_{i}=\beta_{i}=5$

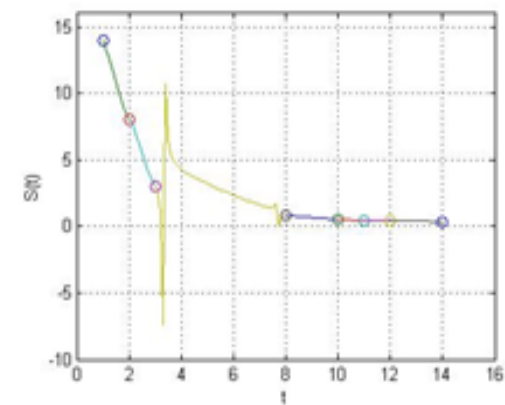

Figure 1(f) : Rational Quadratic Trigonometric Spline interpolation with $\alpha_{i}=\beta_{i}=-1$

A positive data set in table (I) is taken from [9]. Figure 1(a) is generated by using cubic Hermite spline scheme through data set taken in table I. It is noted that figure 1(a) does not preserve the shape of positive data. Figure 1(b) and 1(c) are drawn by using the scheme developed in [9]. Figure 1(d) and 1(e) are drawn by using the scheme developed in section 4 to preserve the positivity preserving everywhere with the values of free parameters set as $\alpha_{i}=\beta_{i}=.1$ and $\alpha_{i}=\beta_{i}=.5$ respectively. Figure 1(e) is more pleasant and smooth as compared to figure 1(d). The scheme does not allow the negative value of negative values of shape parameters as shown in figure 1(f).

Table II: Positive Data Set

\begin{tabular}{|c|c|c|c|c|c|c|c|}
\hline $\mathrm{i}$ & 1 & 2 & 3 & 4 & 5 & 6 & 7 \\
\hline$t_{i}$ & 2 & 3 & 7 & 8 & 9 & 13 & 14 \\
\hline$f_{i}$ & 10 & 2 & 3 & 7 & 2 & 3 & 10 \\
\hline
\end{tabular}

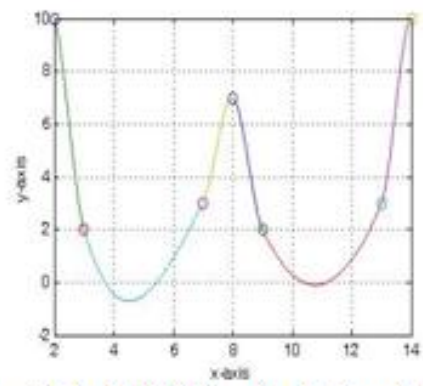

Figure 2(a): Cubic Hermite Spline Interpolation

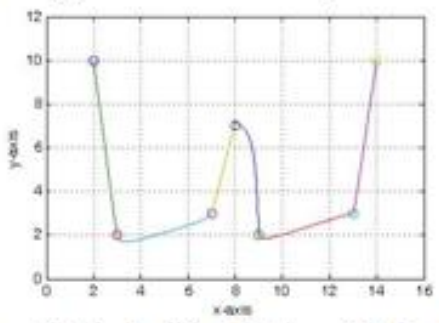

Figure 2(b) : Positive Rational Cubic Spline [9] with $\eta_{\mathrm{l}}=w_{i}=.1$ 


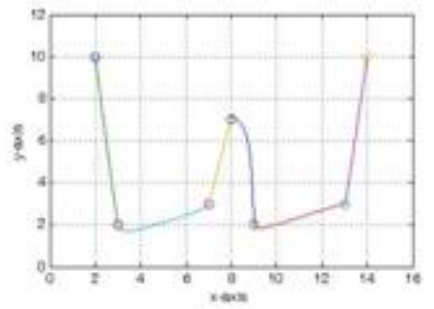

Figure 2(c) : Positive Rational Cubic Spline [9] with $r_{i}=w_{i}=.5$

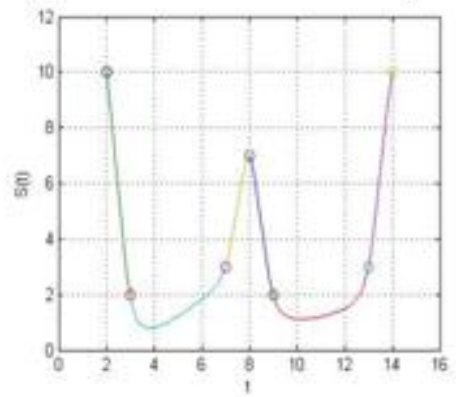

Figure 2(d): Rational Quadratic Trigonometric Spline interpolation with $\alpha_{i}=\beta_{i}=1$

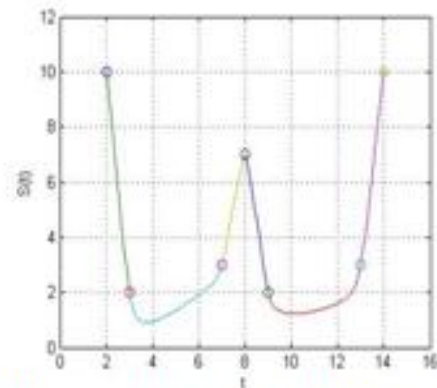

Figure 2(e): Rational Quadratic Trigonometric Spline interpolation with $\alpha_{i}=\beta_{i}=.5$

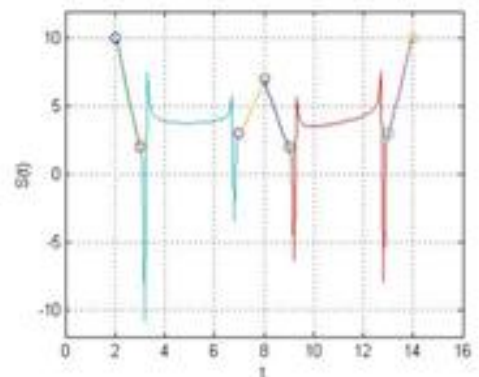

Figure 2(f): Rational Quadratic Trigonometric Spline interpol ation with $\alpha_{i}=\beta_{i}=-.1$

A positive data set in table (II) is borrowed from [9]. The curve in figure 2(a) is drawn by cubic Hermite spline scheme through positive data. It is clear from figure 2(a) that does not preserve the shape of positive data. Figure 2(b) and 2(c) are developed by scheme in [9]. Figure 2(d) and 2(e) are drawn by using the scheme developed in section 4 . To preserve the shape of positive data with the values of shape parameters set as $\alpha_{i}=\beta_{i}=.1$ and $\alpha_{i}=\beta_{i}=.5$ respectively. Figure 2(e) is more pleasant as compare to figure 2(d). The scheme does not allow the negative values to the shape parameters as shown in figure 2(f).

\section{Conclusion}

A $C^{1}$ piecewise rational quadratic trigonometric spline interpolation with three shape parameters has been developed for the positive preserving of positive data to overcome the difficulty of the curve when it lost the positivity by using the cubic Hermite spline scheme. Data dependent shape constraints are derived for single shape parameter to assure the positive shape preservation of the data while other two are left for users choice to refine the curve as desired. The choice of arithmetic mean has been adopted for derivative computation. But in general choice of derivative parameters are left at the wish of users as well. Any numerical derivatives like 
arithmetic, geometric or harmonic mean choice can be used. The developed scheme has following advantageous over existing scheme. It produces $C^{1}$ interpolant. No additional knots are inserted. The scheme is computationally economic and time saving as compared to existing schemes.

\section{References}

[1] Butt, S. and Brodlie, K.W., Preserving positivity using piecewise cubic interpolation. Comput. Graphics, vol. 17, no. 1, 1993, pp. 55-64.

[2] Sarfraz, M., Hussain, M.Z. and Nasar, A., Positive data modeling using spline function. Applied Mathematics and Copmputation. 2010, vopl 216, pp. 2036-2049.

[3] Hussain, M.Z. and Ali, J.M., Positivity preserving piecewise rational cubic interpolation. Mathematika, vol, 22,2006, pp. $147-153$.

[4] Gregory, J.A. and Sarfraz, M., A rational cubic spline with tension. Computer Aided Geometric Design, vol. 7, no. 1-4, 1990, pp. 1-13.

[5] Schmidt, J. and $\mathrm{He} \beta$, W., Positivity of cubic polynomial on intervals and positive spline interpolation. BIT 1988, vol. 28, pp. 340352.

[6] Lamberti, P. and Manni, C., Shape preserving $C^{2}$ functional interpolation via parametric cubic.Numerical Algorithms, vol. 28, 2001, pp. 229-254.

[7] Hoschek, J. and Lasser, D., Fundamental of Computer Aided Geometric Design, translated by L.L. Schumaker. Massachusetts: A K Peters, Wellesley. 1993.

[8] Schoenberg, I.J., On trigonometric spline interpolation. J. Math. Mech. 1994, vol. 13, pp. $795-825$.

[9] Han, Xi-An., Yi Chen M., and Huang, X., The cubic trigonometric Bezier curve with two shape parameters. Applied Math. Letters. 2009, vol. 22, pp. 22, 226-231.

[10] Farin, G., Curves and surfaces for Computer Aided Geometric Design: A practical Guide. $5^{\text {th }}$ Edition. San Diego New York: Academic Press, Inc. 1993.

[11] Hussain, M.Z. and Sarfraz, M., Positivity preserving interpolation of positive data by rational cubics. Journal of Comput. and applied Math. 2008, vol. 218: pp. 446-458. 